\title{
Hemosiderotic fibrolipomatous tumor: A poorly known entity
}

Selma Chaieb, Manel Njima, Nouha Ben Abdeljelil, Ahlem Bellalah, Sarah Chouchane, Abdelfatteh Zakhama, Adnen Moussa

Department of Pathology, Fattouma Bourguiba University Hospital, Monastir, Tunisia

Received: March 15, 2018

Accepted: June 24, 2018

Online Published: June 28, 2018

DOI: $10.5430 /$ crcp.v5n $1 \mathrm{p} 23$

URL: https://doi.org/10.5430/crcp.v5n1p23

\begin{abstract}
Hemosiderotic fibrohistiocytic lipomatous tumor (HFLT) is a rare and supposedly benign fibrolipomatous entity. Clinical and imaging features are not specific. So, histologic, immunohistochemical and molecular analyses are required for diagnosis. The clinic and pathologic aspects of this lesion are diversely reported. Moreover, its reactive or neoplastic origin is still under debate. This lesion should be treated by complete excision. It is mandatory to be aware of its tendency to harbor higher-grade lesions with aggressive outcome. We report a case of HFLT affecting the dorsum of the third right finger in a 52-year-old women, in order to describe histologic patterns of this tumor and to discuss the current literature regarding the etiopathogeny and the cytogenetic features of this rare entity.
\end{abstract}

Key Words: Hemosiderotic fibrohistiocytic lipomatous tumor, Fibroblast-like spindle cells, Mature adipocytes, Hemosiderin

\section{INTRODUCTION}

Hemosiderotic fibrohistiocytic lipomatous lesion renamed in nomenclature to hemosiderotic fibrolipomatous tumor (HFLT) is a rare soft tissue lesion characterized by a proliferation of uniform fibroblastic spindle cells and mature adipocytes with prominent hemosiderin deposition. Since the original description in 2000, around 40 cases have emerged in the literature. HFLT predominantly occurs in women in their fifth or sixth decade of life. The usual sites for HFLT are the dorsum of the foot, the ankle and the dorsum of the hand. ${ }^{[1]}$ Its etiology is debated. Previous local trauma may take part, but the neoplastic origin of the lesion is now admitted owing to a typical chromosomal translocation $\mathrm{t}(1 ; 10)(\mathrm{p} 22 ; \mathrm{q} 24){ }^{[2]}$ We report one patient with HFLT, in order to describe the anatomopathologist's key points of this tumor.

\section{CASE PRESEntation}

A 52-year-old women with no significant medical history or trauma of the upper extremity, presented with a mass on the dorsum of the third right finger. The lesion had slowly increased in size during the last five years. Physical examination revealed a $2 \mathrm{~cm}$, mobile, slightly firm and painful mass on the dorsal aspect of the proximal phalanx of the finger. There were no associated symptoms of local skin inflammation. Metacarpo-phalangeal and interphalangeal joint mobility was normal. Preoperative magnetic resonance (MR) imaging revealed a single subcutaneous, predominantly fatty, soft tissue mass, diagnosed as a lipoma. Surgical removal of the mass was recommended. A diagnosis of HFLT was made on histopathological examination of excisional biopsy. Macroscopically, the mass was unencapsulated soft, mobile, and tender. The cut surface was brown yellow composed of

\footnotetext{
*Correspondence: Selma Chaieb; Email: selmachaieb@yahoo.fr; Address: Department of Pathology, Fattouma Bourguiba University Hospital, Monastir, Tunisia.
}

Published by Sciedu Press 
fatty tissue with areas of macroscopic hemorrhage.

As shown in Figure 1, the lesion demonstrated mature adipocytes and foci of spindle cells consistent with fibrohistiocytic cells, dissecting through the fat in fascicles, or in a hon- eycomb fashion, in association with abundant hemosiderin pigment. No atypia was observed. At immunohistochemistry, the spindled cells were positive for CD34 and CD68 and negative for S-100 protein and AML. A diagnosis of HFLT was made.

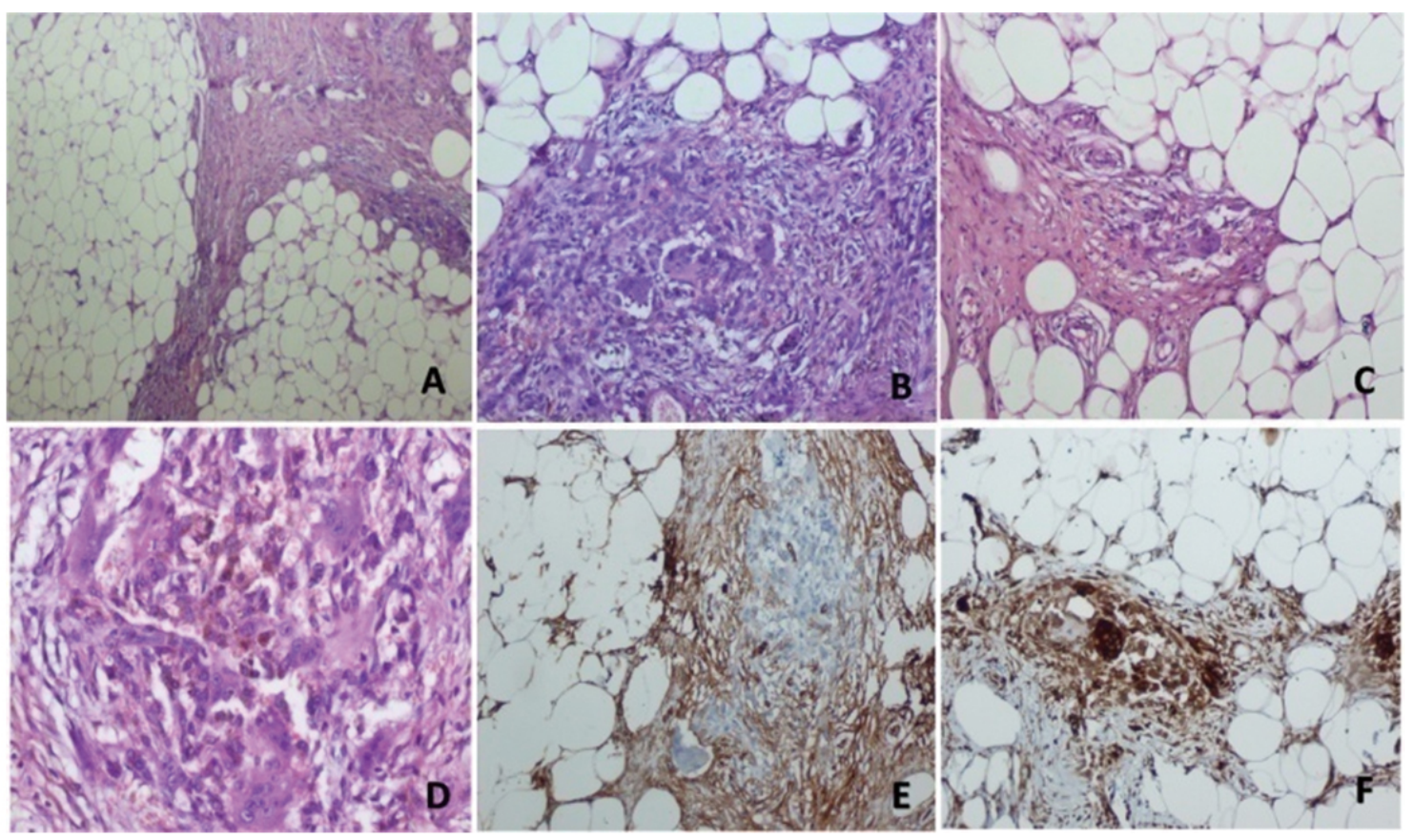

Figure 1. Histopathological findings in a hemosiderotic fibrolipomatous tumor of the dorsum of the third right finger. A) A fatty tumor in the subcutaneous soft tissue composed of lobules of mature adipose tissue separated by vascular connective septa (Stain, hematoxylin and eosin; original magnification, $\times 100)$. B) and C) Bland spindled cells with fibrohistiocytic morphology and osteoclast-like giant cells with scattered clusters of chronic inflammatory cells (Stain, hematoxylin and eosin; original magnification, $\times 200$ ). D) The spindled cells demonstrated vesicular chromatin, small indistinct nucleoli, whithout significant mitotic activity nor cytologic atypia. Abundant macrophage-engulfed hemosiderin (Stain, hematoxylin and eosin; original magnification, $\times 400)$. E) and F) Immunohistochemical analysis: notable staining for CD34 and CD68 of the spindled cells (original magnification, $\times 200$ ).

Complete surgical resection was recommended. The patient was informed on the risk for local recurrence and negligible malignant potential. There were no operative or postoperative complications.

\section{DisCussion}

HFLT may be dermal, dermal-subcutaneous, or subcutaneous in location. It typically presents as a painful, slowly growing, bluish atrophic plaque or mass at the dorsal surface of the ankle/foot in middle-aged adults, with a female predilection. Our case had the appearance of a simple lipoma on clinical examination and MR images. The primary differential diagnosis for HFLT on imaging includes grossly fatty masses as simple lipomas, lipoma variants, atypical lipomas and well-differentiated liposarcomas. ${ }^{[3]}$

Grossly, HFLT is an unencapsulated, yellow-brown fatty tissue tumor with areas of macroscopic hemorrhage. ${ }^{[3]}$

HFLT is a locally aggressive tumor within the range of fibrous lesions involving the subcutaneous tissue. In a small biopsy, it can be confused histologically with spindle-cell lipomas, well differentiated liposarcomas and other atypical lipomatous neoplasms, but it lacks myxoid stroma and the heterogeneous adipocyte component of these lipomatous tumors. ${ }^{[4,5]}$ Other mimickers of HFLT include dermatofibrosarcoma protuberans, plexiform fibrohistiocytic tumors and pleomorphic hyalinizing angiectatic tumor. However, as in our case, the presence of hemosiderin pigment within the lesional cells, the prominence of adipocytes and ectatic hyalinized vessels in HFLT should easily allow this distinction. ${ }^{[3]}$

Limited sampling has the potential for missing a sarcomatous component of the lesion. ${ }^{[6]}$ The association of HFLT with 
myxoinflammatory fibroblastic sarcoma-like tumors, ${ }^{[7,8]}$ or with higher grade myxoid sarcomas, mostly in recurrence following resection has been documented in some reports. ${ }^{[6]}$

The architectural growth pattern is best evaluated in a large representative biopsy which highlights the three main histopathological features in varying proportions: mature adipocytes, a proliferation of uniform fibroblastic spindle cells and hemosiderin pigment. Prominent hemosiderin deposition is variably found in the stroma, in macrophages and in the cytoplasm of spindle cells. ${ }^{[9]}$

Immunohistology is helpful to come to the diagnosis. The spindled cells are positive for CD34 and negative for S-100 protein, caldesmon, desmin and epithelial membrane antigen. These features also were seen in our case. A panel of immunohistochemical markers, including fibrohistiocytic (CD68), vascular (CD34, CD31, Factor XIIIa), neural (S100) and melanocytic markers (HMB-45, Melan-A), can be helpful for differential diagnosis. ${ }^{[6]}$ However, a tumor in superficial acral soft tissue harboring cells containing hemosiderin, a predominant component of mature adipocytes arranged in lobules, with fibrous septae composed of plump, slightly pleomorphic, fibrohistiocytic spindle cells and inflammatory cells, should strongly raise the suspicion of HFLT. ${ }^{[9]}$

The initial hypothesis of a reactive process, based on a history of antecedent local trauma ${ }^{[9]}$ has not been proven. Newer case series revealed clonal translocations between chromosomes 1 and $10(\mathrm{t}(1 ; 10)(\mathrm{p} 31 ; \mathrm{q} 25)$ and $\mathrm{t}(1 ; 10)(\mathrm{p} 22 ; \mathrm{q} 24))$, involving TGFBR3 on chromosome 1q and MGEA5 on chromosome 10q, suggesting that HFLT is neoplastic in origin. Clonal translocations of TGFBR3 and/or MGEA5 genes have also been found in myxoinflammatory fibroblastic sarcoma including cases with mixed features of HFLT and myxoinflammatory fibroblastic sarcoma, suggesting a close relation between these entities. ${ }^{[10]}$ These gene rearrangements have also been identified in pleomorphic hyalinizing angiectatic tumors with histopathological features of HFLT and, less consistently, in classic pleomorphic hyalinizing angiectatic tumors. ${ }^{[1]}$ Furthermore, Antonescu et al. ${ }^{[12]}$ detected the presence of high level amplification of VGLL3 locus on

\section{REFERENCES}

[1] Browne TJ, Fletcher CD. Haemosiderotic fibrolipomatous tumour (so-called haemosiderotic fibrohistiocytic lipomatous tumour): analysis of 13 new cases in support of a distinct entity. Histopathology. 2006; 48: 453-61. PMid:16487368. https://doi.org/10.1111/ j.1365-2559.2006.02360.x

[2] Wettach GR, Boyd LJ, Lawce HJ, et al. Cytogenetic analysis of a 3p.12.1 by FISH in HFLT and myxoinflammatory fibroblastic sarcoma, as well as in cases with mixed morphology, but in none of the three cases of pleomorphic hyalinizing angiectatic tumors. These findings suggest that HFLT is an integral part of the group of mesenchymal tumors with translocation. Molecular analysis may be a significant complementary method in diagnostically challenging cases or in small biopsies.

HFLT has been reported to have a benign clinical course, ${ }^{[3,4]}$ but a locally recurring potential. ${ }^{[5]}$ The homogeneously sized and well-differentiated adipocytes, that are usually the main component of HFLT, have a benign histologic appearance. However, up to $30 \%$ to $50 \%$ local recurrence or residual disease following primary resection were observed on follow up. ${ }^{[1]}$ The high recurrence rate and residual disease appears to be related to partial excisions at the time of surgery. Potential reasons include poor visualization of the margin of the tumor owing to its ill-defined border blended into the normal fat in the majority of cases and the large size of the original lesion. ${ }^{[3]}$ These slowly growing tumors have a generally favorable prognosis. However, malignant transformation and metastases were reported. ${ }^{[5]}$ HFLT has been reported to be prone to malignant transformation into sarcoma of variable histologic grade: myxoid sarcoma, myxoinflammatory fibroblastic sarcoma and high-grade myxofibrosarcoma. ${ }^{[5,10]}$ Patients should be therefore, informed about the risk of recurrence and the need of regular yearly clinical follow up.

In conclusion, HFLT is a slow-growing, subcutaneous, acral soft tissue tumor, with distinctive histopathological features. In agreement with the few previous reports, ${ }^{[3-5]}$ the diagnosis of HFLT was made, in our case on morphological and immunohistochemical characteristics. Immunohistochemistry and molecular genetics investigations can help to come to the diagnosis and eventually to predict the outcome of patients, especially in hybrid tumors. Owing to its malignant potential, HFLT should be treated by large resection and close follow-up.

\section{CONFLicts of InTEREST Disclosure}

No conflicts of interest. hemosiderotic fibrolipomatous tumor. Cancer Genet Cytogenet. 2008; 15: 140-3. PMid:18406878. https://doi.org/10.1016/j.canc ergencyto.2008.01.012

[3] Moretti VM, Brooks JS, Ogilvie CM. Hemosiderotic Fibrohistiocytic Lipomatous Lesion. A Clinicopathologic Characterization. Clin Orthop Relat Res. 2010; 468: 2808-13. PMid:20127213. https: //doi.org/10.1007/s11999-010-1242-7 
[4] Wilk M, Zelger BG, Zelger B. Hemosiderotic Fibrolipomatous Tumor. Am J Dermatopathol. 2016; 38(9): 714-6. PMid:26844619. https://doi.org/10.1097/DAD.0000000000000540

[5] Solomon DA, Antonescu CR, Link TM, et al. Hemosiderotic Fibrolipomatous Tumor, Not an Entirely Benign Entity. Am J Surg Pathol. 2013; 37(10): 1627-30. PMid:24025526. https://doi.or g/10.1097/PAS. 0b013e31829ff078

[6] Morency E, Laskin W, Lin X. Cytologic and Histologic Features of Pleomorphic Undifferentiated Sarcoma Arising in a Hybrid Hemosiderotic Fibrolipomatous Tumor and Pleomorphic Hyalinizing Angiectatic Tumor: Report of an Unusual Case with a Literature Review. Acta Cytol. 2015; 59(6): 493-7. PMid:26841226. https://doi.org/10.1159/000443319

[7] Elco CP, Marino-Enriquez A, Abraham JA, et al. Hybrid myxoinflammatory fibroblastic sarcoma/hemosiderotic fibrolipomatous tumor: report of a case providing further evidence for a pathogenetic link. Am J Surg Pathol. 2010; 34: 1723-7. https://doi.org/10.109 7/PAS . Ob013e3181f17d51

[8] Zreik RT, Carter JM, Sukov WR, et al. Myxoinflammatory fibroblastic sarcoma (MIFS) and hybrid hemosiderotic fibrolipomatous tumor (HFLT)/MIFS: related or not? Mod Pathol. 2015; 28(suppl 2): 30A.
[9] Marshall-Taylor C, Fanburg-Smith JC. Hemosiderotic fibrohistiocytic lipomatous lesion: ten cases of a previously undescribed fatty lesion of the foot/ankle. Mod Pathol. 2000; 13: 1192-9. PMid:11106076. https://doi.org/10.1038/modpathol.3880221

[10] Zreik RT, Carter JM, Sukov WR, et al. TGFBR3 and MGEA5 rearrangements are much more common in "hybrid" hemosiderotic fibrolipomatous tumor-myxoinflammatory fibroblastic sarcomas than in classical myxoinflammatory fibroblastic sarcomas: a morphological and fluorescence in situ hybridization study. Hum Pathol. 2016 Jul; 53: 14-24.

[11] Carter JM, Sukov WR, Montgomery E, et al. TGFBR3 and MGEA5 rearrangements in pleomorphic hyalinizing angiectatic tumors and the spectrum of related neoplasms. Am J Surg Pathol. 2014; 38(9): 1182-992. PMid:24705316. https ://doi.org/10.1097/PAS . 00 00000000000212

[12] Antonescu CR, Zhang L, Nielsen GP, et al. Consistent $\mathrm{t}(1 ; 10)$ with rearrangements of TGFBR3 and MGEA5 in both myxoinflammatory fibroblastic sarcoma and hemosiderotic fibrolipomatous tumor. Genes Chromosomes Cancer. 2011 Oct; 50(10): 757-64. https://doi.org/10.1002/gcc. 20897 